\title{
Ética y Jurisprudencia Administrativa de los Derechos de los Sujetos de Investigación en Pandemia (COVID-19): Función de los Comités Éticos Científicos: Chile
}

\author{
Ethics and Administrative Jurisprudence of the Rights of Subjects of Research \\ in Pandemic (COVID-19): Role of the Scientific Ethics Committees: Chile
}

\author{
Omar Espinoza-Navarro ${ }^{1}$ \& Sebastián Rivera Gutiérrez ${ }^{2}$
}

ESPINOZA-NAVARRO, O. \& RIVERA, G. S. Ética y jurisprudencia administrativa de los derechos de los sujetos de investigación en pandemia (COVID-19): función de los comités éticos científicos: Chile. Int. J. Morphol., 39(3):785-788, 2021.

RESUMEN: Todo gobierno debe reaccionar rápida y efectivamente ante cualquier pandemia, Chile no es la excepción y apoyado en el estado de Excepción Constitucional, ha tenido que implementar medidas que podrían involucrar poca información sobre las percepciones de las personas y las reacciones durante la implementación de las restricciones. Las instituciones internacionales de salud han determinado que es un deber moral realizar investigaciones que generen evidencia que promuevan y mejoren la atención de la salud y la mitigación de la pandemia, instando a reducir los "obstáculos" prácticos de la revisión ética. Los objetivos de este trabajo fueron analizar desde las perspectivas de las consideraciones éticas y jurídicas, el rol que cumplen los Comités Éticos Científicos en el manejo y la protección de las personas durante la pandemia de la COVID-19. La metodología de trabajo se basó en la recolección de la información de Instituciones nacionales e internacionales de Salud y luego analizarla según la jurisprudencia administrativa del gobierno de Chile. Se concluye que los cambios de criterios que deben observar los CECs en el proceso de revisión de los protocolos de los proyectos de investigación científica, deben velar por proteger los derechos de los pacientes y sujetos de investigación en cuanto puede involucrar información sensible, más aún, si se consideran las graves consecuencias de su transgresión, dar un sentido distinto al que corresponda a las normas sobre derechos de pacientes, puede resultar en "falta de servicio" y eventual vulneración en los derechos del sujeto de investigación. La labor de los CEC, debe realizarse siempre desde una interpretación restrictiva, reconociendo la función pública que cumplen como parte integrante de la labor ética encomendada por el legislador al efecto.

PALABRAS ClAVE: Ministerio de Salud; Aceptabilidad Ética; Manejo en Pandemia; COVID-19; Chile.

\section{INTRODUCCIÓN}

La Comisión Belmont definió en el año 1979, las condiciones a cumplir para la protección de los sujetos humanos en la investigación biomédica, basado en sólidos principios de respeto a las personas, beneficencia y justicia. Sin embargo en la actualidad se plantea la necesidad de incluir otros principios éticos, como integridad científica y fidelidad, principios que se caracterizan por un compromiso con la veracidad, la responsabilidad y adherencia en el cumplimiento de los compromisos y de buena conducta profesional (Warner \& Weiss, 2004).

La investigación científica biomédica es una práctica donde se manifiesta la capacidad del ser humano, para explorar, exáminar, plantear nuevos objetivos y vencer las dificultades, es un proceso sistemático, organizado que se supone permitirán beneficios para los individuos y para la sociedad, asegurando la permanente necesidad de progresos y avances en todos los campos de la medicina. Sin embargo los grandes escándalos y tragedias cometidos en la investigación con humanos, concluyeron que este tipo de investigación requiere un gran compromiso de responsabilidad de los Comités Éticos Científicos, que resguarden en las revisiones de los protocolos de investigación, la transparencia, derechos a retiro, protección de grupos vulnerables, privacidad y declaración de daños (O`Ryan, 2017).

Los diferentes efectos de la pandemia COVID-19 y posiblemente de todas las futuras pandemias, impactan en las políticas gubernamentales y de las Instituciones regulatorias nacionales e internacionales, con anuncios de reemplazo o suspensión de reglas de protección para pacientes, con la intención de acelerar ensayos clínicosque están

\footnotetext{
${ }^{1}$ Departamento de Biología, Facultad de Ciencias, Comité Ético Científico, Universidad de Tarapacá, Chile.

${ }^{2}$ Dirección de Asuntos Legales, Representante Jurídico del Comité Ético Científico, Universidad de Tarapacá, Chile.
} 
en curso en algunas de sus fases intermedias. Apelando al sentido de urgencia pandémica, se implementan medidas, se suspenden derechos constitucionales y se flexibilizan mecanismos de control, soluciones que se suponen son transitorias. El 13 de julio de 2020 se presenta en la Cámara de Diputados de Chile una moción parlamentaria para modificar el "Código Sanitario", permitiendo que en caso de alerta sanitaria, se puedan autorizar ensayos clínicos con reglas diferentes a las originales, lo cual incluso altera el marco legal de la Ley 20.850 (Ricarte Soto), que configura una fuerte protección a los pacientes que participan en estos ensayos, dejándoles entonces, sin protección en estas alertas sanitarias.

Chile en sus políticas de manejo y control de la pandemia de la COVID-19, debe también fortalecer el rol de los Comités Éticos Científicos en sus conceptos éticos, científicos, sociales y jurídicos, en la defensa de los pacientes o participantes voluntarios en las investigaciones biomédicas (Herrera-Medina \& Espinoza-Navarro, 2020). El objetivo de este estudiofue reflexionar sobre el rol de los Comités Éticos Científicos en estos tiempos de "excepción" en la protección de los sujetos de investigación en losaspectos éticos - valóricos y de la jurisprudencia administrativa.

\section{MATERIAL Y MÉTODO}

La metodología de trabajo se basó en la recolección de la información utilizando la plataforma de Informática del Sistema Público de Chile, con acceso directo y completo a través de la ley de transparencia, además de la información disponible de los sistemas de PubMed y Medline y de los Organismos Nacionales e Internacionales de ética y de salud. La información recopilada se analizó según sus aspectos éticos y de la jurisprudencia del gobierno de Chile.

Aspectos éticos valóricos. En situaciones de pandemia la investigación científica es fundamental, es un deber ético y un gran desafíopara los investigadores que deben generar conocimiento rápido para superar los problemas sanitarios, sociales y económicos relacionados. Sin embargo, para resguardar los aspectos éticos-valóricos y jurídicos en los protocoles de investigación, un grancometido tendrán los Comités Éticos Científicos (CECs). Al respecto las normativas internacionales de las "Buenas Prácticas Clínicas" (BPC), representan los estándares internacionales de principios éticos y de calidad de la investigación, dirigidos a proteger los derechos, la seguridad y el bienestar de los sujetos del estudio y que deben prevalecer sobre los intereses de la Sociedad y de la Ciencia. Sus protocolos establecen que los riesgos potenciales a los sujetos se deben minimizar, potenciando los beneficios y determinan que los estudios deben realizarse de acuerdo con protocolos ya aprobados por un Comité Ético Científico Independiente. Ningún estudio puede iniciarse sin que previamente se haya obtenido esta aprobación. Todo consentimiento informado voluntario de cada uno de los sujetos del estudio, debe ser acordado antes del inicio de la investigación y de la participación del sujeto.Las personas responsables de los estudios deben tener la educación, el entrenamiento y la experiencia adecuada, para participar y cumplir con las responsabilidades que se le asignen en el estudio clínico.

Las pautas de BPC son utilizados en ensayos clínicos en todo el mundo con el objetivo principal de proteger y preservar los derechos humanos (EUR-Lex, 2001). La legislación chilena en la Resolución N ${ }^{\circ} 460$, del Instituto de Salud Pública, Ministerio de Salud del 20 de enero de 2015 (Instituto de Salud Pública, 2015), menciona la obligación de aplicar las normas de BPC en la planificación, realización, registro y comunicación de todos los ensayos clínicos que se realicen en el País (EUR-Lex; World Health Organization, 2002; Vijayananthan \& Nawawi, 2008; Instituto de Salud Pública).

Debido a los devastadores efectos de la pandemia de la COVID-19, las instituciones internacionales de salud, han determinado que es un deber moral realizar investigaciones que generen evidencia que promuevan y mejoren la atención de la salud y mitigación de la pandemia y se pide reducir los obstáculos prácticos de revisión ética. La Organización Panamericana de la Salud en continuos reportes, aporta con orientaciones y estrategias para agilizar la revisión y supervisión ética de las investigaciones relacionadas (2020). Los organismos chilenos de Salud, basándose enel Decreto de Estado de Excepción Constitucional de Catástrofe (2020), también orienta sobre los nuevos procesos en la obtención de Consentimiento Informado y el enrolamiento de los pacientes en contexto de pandemia (Organización Panamericana de la Salud, 2020; Ministerio de Salud, 2020).

Aspectos de Jurisprudencia Administrativa (desde la función de los CEC). La jurisprudencia administrativa sobre derechos del sujeto de investigación, desde la perspectiva de la función de los Comités Éticos Científicos, establece la extensión de la autonomía de los C.E.C., para la interpretación normativa de su función, teniendo en consideración, que los citados entes colegiados, si bien se encuentran dotados de existencia legal, en la gran mayoría de los casos, forman parte de entidades públicas (hospitales y universidades por ejemplo), las cuales carecen de autonomía plena para interpretar las normas legales que regulan los derechos del sujeto de investigación, por lo que, dar un sentido y alcance distinto al que corresponda a las normas, sobre derechos de los pacientes, puede desviar el sentido en que se interpretan las normas jurídicas que regulan la materia, circunstancia que en nuestro Estado de Derecho, se puede considerar como "falta de servi- 
cio", concurriendo en una eventual vulneración en los derechos del sujeto de investigación.

En este marco normativo, los C.E.C., tienen por responsabilidad esencial el proteger los derechos, la seguridad y el bienestar de los sujetos de investigaciones realizadas en el contexto de la ley $\mathrm{N}^{\circ} 20.120$ (Ministerio de Salud, 2006) y su Reglamento, debiendo evaluar proyectos de investigaciones científico-médicas que sean sometidas a su consideración. Sin embargo, al encontrarse insertos en entidades que forman parte de la Administración Pública, su esfera de deberes alcanza un rango legal superior, ya que, conforme a la Constitución Política de la República de Chile (2005) (art. $1^{\circ}$ ) y a la Ley Orgánica Constitucional de Bases Generales de Administración del Estado, Nº 18.575 (art. 3) (Ministerio del Interior, 1986), la Administración del Estado está al servicio de la persona humana y su finalidad es promover el bien común atendiendo las necesidades públicas en forma continua y permanente.

Conforme a lo anteriormente expuesto, en el Dictamen $\mathrm{N}^{\circ} 8.113$, emitido el 20 de abril de 2020, la Contraloría General de la República (2020), abordó el tema de la confidencialidad de los derechos del paciente, desde lo dispuesto en el artículo $19 \mathrm{~N}^{\circ} 4$ de la Constitución Política de la República de Chile (2005), que asegura a todas las personas el respeto y protección a la vida privada, a la honra de la persona y su familia, y la protección de sus datos personales; estableciendo que el tratamiento y protección de estos datos se efectuará en la forma y condiciones que determine la ley (Ley 20.584, Ministerio de Salud, 2012) y razona, que la información de salud relativa a los pacientes que hayan sido diagnosticados con COVID-19, forma parte de la ficha clínica de cada uno de ellos y en consecuencia, constituye un dato sensible que solo puede ser objeto de tratamiento, cuando la ley lo autoriza expresamente.

Cabe preguntarse entonces, ¿cómo incide este pronunciamiento en el funcionamiento de los C.E.C.?Al respecto al dictamen $\mathrm{N}^{\circ} 52.957 / 2016$, de la Contraloría General de la República (2016), sobre el requerimiento realizado por el C.E.C. del Hospital Naval Almirante Nef, en relación a si, conforme a lo dispuesto en la ley $\mathrm{N}^{\circ} 20.120$ resultaba posible a esa entidad colegiada, aprobar "investigaciones científicas retrospectivas", en las que los respectivos científicos necesiten utilizar datos biomédicos anónimos obtenidos de las fichas clínicas de pacientes, sin contar con el consentimiento de estos, y ante el vacío legal, la entidad superior de control, estableció en su pronunciamiento, que - esta materia debe resolverse conforme a lo dispuesto en la ley $\mathrm{N}^{\circ} 20.584$, que regula los derechos y deberes que tienen las personas en relación con acciones vinculadas a su atención en salud- y no conforme a la ley $\mathrm{N}^{\circ} 20.120$, razonando que "la ley no autoriza a que los científicos, a fin de efectuar estudios biomédicos retrospectivos, accedan a la ficha clínica de determinada persona, a no ser que cuenten con el consentimiento expreso de esta, por tratarse de datos sensibles, ni aun con el objeto de que estos sean disociados". Este pronunciamiento inmediatamente impactó en las investigaciones y en los derechos de los pacientes. Extrapolándose nuevamente el mismo criterio al año 2020, con ocasión de la publicación de los datos de las personas diagnosticadas COVID-19 en el marco de sus derechos.

\section{DISCUSIÓN}

Muchos desafíos se presentarán en el futuro cercano debido al avance vertiginoso de la ciencia y la tecnología. Los Comités Éticos Científicos y la Sociedad misma, deberán velar paraque los valores éticos y jurídicos se respeten y se actualicen al ritmo acelerado de estos cambios, de tal manera de aprovechar la investigación biomédica internacional "con seres humanos" en mejorar las atenciones en salud pública, favoreciendo la justicia distributiva y la justicia social (Páez Moreno, 2012; Ministerio de Salud, 2020). No obstante la optimización de los tiempos de respuesta, se debe garantizar siempre, la rigurosidad de la revisión de los protocolos por parte de los Comités Éticos Científicos en un trabajo conjunto con los investigadores (Jamrozik et al., 2021). Trabajos realizados por Bull et al. (2020), determinan que en los nuevos estudios sobre COVID-19, (estudios de infección humana controlada, CHI, por sus siglas en inglés), deben prevalecer siempre, la minimización de los riesgos en todos los pacientes o voluntarios participantes, con evaluaciones contínuas y sistemáticas de riesgos.

Durante los tiempos de pandemia o crisis sanitaria los CECs deberán facilitar la ejecución en forma rápida de proyectos de investigación orientados a la prevención y manejo de la pandemia, pero velando por la protección de los pacientes en ensayos clínicos. Las instituciones públicas y privadas en un esfuerzo mancomunado deben incentivar toda la capacidad científica y tecnológica para comprender estos fenómenos que podrían repetirse en el futuro (Jamrozik et al.).

En conclusión, los cambios de criterios que deben observar los CEC en el proceso de revisión de los proyectos de investigación científica en tiempos de pandemia, deben velar por proteger los derechos de los pacientes y sujetos de investigación en cuanto puede involucrar información sensible, más aún, si se consideran las graves consecuencias de su transgresión, conforme a la normativa penal y sanitaria vigentey conforme ello, queda demostrado que la labor de los CEC, debe realizarse siempre desde una interpretación restrictiva, reconociendo la función pública que cumplen como parte integrante de la labor ética encomendada por el legislador al efecto. 
AGRADECIMIENTOS. Este trabajo contó con el auspicio del proyecto UTA Mayor $\mathrm{N}^{\circ} 4716-17$, Universidad de Tarapacá, Arica-Chile.

Los autores declaran que no tiene vinculación con empresa o laboratorios farmaceúticos, ni conflictos de intereses relacionados con producción y ventas de vacunas. Nuestra posición no representa necesariamente la opinión oficial de la Universidad de Tarapacá, de la cual somos funcionarios.

ESPINOZA-NAVARRO, O. \& RIVERA, G. S. Ethics and administrative jurisprudence of the rights of subjects of research in pandemic (COVID-19): role of Scientific Ethics Committees: Chile. Int. J. Morphol., 39(3):785-788, 2021.

SUMMARY: Every government must react quickly and effectively to any pandemic, Chile is no exception and supported by the state of Constitutional Exception, it has had to implement measures that could involve little information about people's perceptions and reactions during the implementation of the restrictions. International health institutions have determined that it is a moral duty to carry out research that generates evidence that promotes and improves health care and the mitigation of the pandemic, urging to reduce the practical "obstacles" to ethical review. The objective of this study was to analyze from the perspectives of ethical and legal considerations, the role that Scientific Ethics Committees play in the management and protection of people during the COVID-19 pandemic. The methodology used was based on collecting information from national and international Health Institutions and then analyzing it according to the administrative jurisprudence of the Chilean government. It is concluded that the changes in criteria that the CECs must observe in the process of reviewing the protocols of scientific research projects, must ensure the protection of the rights of patients and research subjects insofar as it may involve sensitive information, even more if the serious consequences of its transgression are considered. Giving a different meaning to the one that corresponds may result in "lack of service" and eventual violation of the rights of the research subject. The task of the CEC, must always be carried out from a restrictive interpretation, recognizing the public function that they fulfill as an integral part of the ethical work entrusted by the legislators to that effect.

KEY WORDS: Ministry of Health; Ethical Acceptability; Pandemic Management; Chile.

\section{REFERENCIAS BIBLIOGRÁFICAS}

Bull, S.; Jamrozik, E.; Binik, A. \& Parker, M. J. SARS-CoV-2 challenge studies: ethics and risk minimisation. J. Med. Ethics, 2020. DOI: https://www.doi.org/ 10.1136/medethics-2020-106504

Constitución Política de la República de Chile. Artículos $1^{\circ}, 19$ N $^{\circ} 4$. Santiago de Chile. Constitución Política de la República de Chile, 2005. Disponible en: http://www.bcn.cl

Contraloría General de la República. Dictamen N 52.957/2016. Santiago de Chile, Contraloría General de la República, 2016. Disponible en: http:// www.contraloria.cl/LegisJuri/DictamenesGeneralesMunicipales.nsf/ FormImpresionDictamen?OpenForm\&UNID=0486B386B4CBA70084257FF700441652
Contraloría General de la República. Dictamen N 8.113/2020. Santiago de Chile, Contraloría General de la República, 2020. Disponible en: https:// www.contraloria.cl/pdfbuscador/dictamenes/008113N20/html

EUR-Lex (EPD). Directiva 2001/20/CE del Parlamento Europeo y del Consejo, de 4 de abril de 2001, relativa a la aproximación de las disposiciones legales, reglamentarias y administrativas de los Estados miembros sobre la aplicación de buenas prácticas clínicas en la realización de ensayos clínicos de medicamentos de uso humano. EUR-Lex 2001/20/CE, 2001.

Herrera-Medina, N. \& Espinoza-Navarro, O. Scientific ethical committees and the review of projects in health, COVID-19. Int. J. Odontostomat., 14(4):4667, 2020.

Instituto de Salud Pública. Resolución $N^{\circ} 460$. Santiago de Chile, Ministerio de Salud, Gobierno de Chile, 2015.

Jamrozik, E.; Littler, K.; Bull, S.; Emerson, C.; Kang G.; Kapulu, M.; Rey, E.; Saenz, C.; Shah, S.; Smith, P. G.; et al. Key criteria for the ethical acceptability of COVID-19 human challenge studies: Report of a WHO Working Group. Vaccine, 39(4):633-40, 2021.

Ministerio de Salud. Comité Ministerial de Ética en Investigación en Salud. Recomendaciones para los Comités de Ética Científicos COVID-19. 6 de junio de 2020. Santiago de Chile, Ministerio de Salud, de Chile, 2020.

Ministerio de Salud. Ley 20.120. Sobre la Investigación Científica en el Ser Humano, su Genoma y Prohíbe la Clonación Humana. Artículos 1 y 10. Santiago de Chile, Ministerio de Salud, Subsecretaría de Salud Pública, Gobierno de Chile, 2006. Disponible en: https://www.bcn.cl/leychile/ navegar?idNorma $=253478$

Ministerio de Salud. Ley 20.584. Regula los Derechos y Deberes que tienen las Personas en Relación con Acciones Vinculadas a su Atención en Salud. Santiago de Chile, Ministerio de Salud, Subsecretaría de Salud Pública, Gobierno de Chile, 2012. Disponible en: https://www.bcn.cl/leychile/ navegar?idNorma $=1039348$

Ministerio del Interior. Ley $N^{\circ} 18.575$ Orgánica Constitucional de Bases Generales de Administración del Estado, Artículo $3^{\circ}$. Santiago de Chile, Ministerio del Interior, Subsecretaría del Interior, Gobierno de Chile, 1986. Disponible en: https://www.bcn.cl/leychile/navegar?idNorma=29967

O’Ryan, M. Revisión Metodológica por los Comités Ético Científicos (CEC). XXII Taller de Bioética organizado por el Comité Asesor de Bioética FONDECYT - CONICYT. Santiago de Chile, FONDECYT - CONICYT, 2017.

Organización Panamericana de la Salud (OPS). Orientación y Estrategias para Agilizar la Revisión y Supervisión Éticas de Investigaciones Relacionadas con COVID-19. 7 abril de 2020. Washington (D.C.), Organización Panamericana de la Salud, 2020.

Páez Moreno, R. La investigación internacional en seres humanos: de la justicia distributiva a la justicia social. Rev. Bioética Derecho, (24):20-30, 2012.

Vijayananthan, A. \& Nawawi, O. The importance of Good Clinical Practice guidelines and its role in clinical trials. Biomed. Imaging Interv. J., 4(1):e5, 2008.

World Health Organization (WHO). Good Clinical Practice Guidelines. Ginebra, World Health Organization, 2002.

\author{
Dirección para correspondencia: \\ Omar Espinoza Navarro \\ Departamento de Biología \\ Facultad de Ciencias \\ Comité Ético Científico \\ Universidad de Tarapacá \\ Arica - CHILE
}

\section{E-mail: oespinoz@academicos.uta.cl}

Recibido : 20-02-2021

Aceptado: 02-04-2021 